「材料」(Journal of the Society of Materials Science, Japan), Vol. 64, No. 1, pp. 47-51, Jan. 2015

Original Papers

\title{
Nonlinear Viscoelasticity of Melts of High Density Polyethylenes ${ }^{\dagger}$
}

by

\author{
Takumi KABEYA $^{*}$, Katsuyuki YoshiKAWA ${ }^{* *}$, Jun-ichi HorinAKA ${ }^{* * *}$ and Toshikazu TAKIGAWA ${ }^{* * *}$
}

Damping behavior is investigated for five types of high density polyethylene (HDPE) samples with broader molecular weight distributions. The melt of moderately entangled HDPE shows a weaker damping than the Doi-Edwards (DE) prediction, maybe due to a broader molecular weight distribution. On the other hand, the highly entangled HDPE melts with broader molecular weight distributions show stronger damping than the DE prediction, as in the case of highly entangled systems with narrow molecular weight distributions. The strong damping in the former case appears different in origin from the latter.

\section{Key words:}

Damping function, Highly entangled polymer, Relaxation modulus, Doi-Edwards theory

\section{Introduction}

The entanglement coupling is a unique and most important molecular interaction in melts and concentrated solutions of polymers. The molecular weight between entanglements $\left(M_{\mathrm{e}}\right)$ is a measure of how intensive the interaction works in the system, and the quantity $M_{\mathrm{e}}$ in the molten state is a material constant but even now what controls the polymer-species dependence of $M_{\mathrm{e}}$ appears to be still unclear. Probably, the smallest value of $M_{\mathrm{e}}$ in the molten state is 1,250 for polyethylene $(\mathrm{PE})^{1)}$. This suggests that highly entangled systems are easily obtained if $\mathrm{PE}$ is employed. Especially, as high density polyethylene (HDPE) is known to be a linear polymer, highly entangled linear chain systems are attained by using HDPE.

Understanding entanglement has been one of the main topics in the polymer rheology, and many experimental as well as theoretical studies have been made to clarify the essence of entanglement ${ }^{2}$. We know at present that the outline of the rheology of entangled polymers is now described by the Doi-Edwards (DE) theory ${ }^{3}$. The nonlinear viscoelasticity for the entangled systems is often discussed in terms of a damping function $h(\gamma)$, with $\gamma$ being the strain. To estimate $h(\gamma)$ the relaxation modulus $G(t, \gamma)$ is obtained by the stress relaxation experiments, where $t$ is the elapsed time after the imposition of stepwise strain. In the long time region, $G(t$, $\gamma)$ can be written as a factorized form:

$$
G(t, \gamma)=G(t) h(\gamma)
$$

Here, $G(t)$ is the linear relaxation modulus. If this separability holds, each $G(t, \gamma)$ curve at different $\gamma$ can be superimposed onto $G(t)$ by a vertical shift corresponding to $h(\gamma)$. The DE theory predicts a universal form of $h(\gamma)$ for the entangled polymer systems, and many experimental results really coincide well with the prediction. The damping behavior of these systems is classified as Type A by Osaki ${ }^{4}$. However, there appear to be exceptions; for example, $h(\gamma)$ for highly entangled polymers with narrow molecular weight distribution (mostly, polystyrenes by experiments) deviates from the DE prediction and shows strong strain softening (damping function of Type-C by Osaki ${ }^{4}$ ). Why this strong softening occurs appears to be still unclear, although Marrucci and Grizzuti ${ }^{5)}$ have mentioned a possible origin for the strong softening. More experiments, preferably using other than polystyrenes, should be needed to clarify this peculiar behavior. The aim of this study is to investigate the damping behavior of highly entangled HDPEs.

\section{Experimental}

Three commercially available HDPEs (Asahi Kasei Chemicals Co.) and two HDPE samples, the precursors to commercial products sample-coded as PE-A and PE-B (Japan Polyethylene Co.), were used for rheological measurements. Weight-average molecular weight $\left(M_{\mathrm{w}}\right)$, number-average molecular weight $\left(M_{\mathrm{n}}\right)$ and the ratio $M_{\mathrm{w}} / M_{\mathrm{n}}$ for the PE

Table 1 Number average molecular weight $\left(M_{\mathrm{n}}\right)$, weight average molecular weight $\left(M_{\mathrm{w}}\right)$, the ratio of $M_{\mathrm{w}}$ to $M_{\mathrm{n}}$, and the number of entanglements per chain $\left(n_{\mathrm{e}}\right)$ calculated by $n_{\mathrm{e}}=M_{\mathrm{w}} / M_{\mathrm{e}}$ with $M_{\mathrm{e}}$ being 1250 .

\begin{tabular}{ccccc}
\hline Sample & $M_{\mathrm{n}}$ & $M_{\mathrm{w}}$ & $M_{\mathrm{w}} / M_{\mathrm{n}}$ & $n_{\mathrm{e}}$ \\
\hline T701A & $1.3 \times 10^{4}$ & $5.0 \times 10^{4}$ & 3.9 & 40 \\
SH810 & $4.5 \times 10^{4}$ & $1.8 \times 10^{5}$ & 4.0 & 140 \\
UH850 & $3.0 \times 10^{5}$ & $1.4 \times 10^{6}$ & 4.7 & 1,100 \\
PE-A & $7.3 \times 10^{4}$ & $4.3 \times 10^{5}$ & 5.9 & 340 \\
PE-B & $5.5 \times 10^{4}$ & $3.4 \times 10^{5}$ & 6.2 & 270 \\
\hline
\end{tabular}

$\dagger$ Received May 29, 2014 (C) 2015 The Society of Materials Science, Japan

* Dept. Mater. Chem., Kyoto Univ., Nishikyo-ku, Kyoto, 615-8510 Japan

** Hosokawa Yoko Co. Ltd. Chiyoda-ku, Tokyo, 102-0084 Japan

*** Member : Dept. Mater. Chem., Kyoto Univ., Nishikyo-ku, Kyoto, 615-8510 Japan 
samples are summarized in Table 1 . The average number of entanglements per chain $\left(n_{\mathrm{e}}\right)$, which should be determined in principle by $n_{\mathrm{e}}=M_{\mathrm{n}} / M_{\mathrm{e}}$ but here $n_{\mathrm{e}}$ calculated by $n_{\mathrm{e}}=M_{\mathrm{w}} /$ $M_{\mathrm{e}}$, is listed in the table. Here, $M_{\mathrm{e}}$ was assumed to be 1250 for PE. Disk-shaped specimens for rheological measurements were made by compression molding at $150{ }^{\circ} \mathrm{C}$ for $20 \sim 30$ $\min$.

Dynamic viscoelasticity and stress relaxation (SR) experiments were made by using an ARES rheometer (TA Instruments, USA) with a cone-plate (CP) geometry $(0.1 \mathrm{rad}$ and $25 \mathrm{~mm} \phi$ ) or a parallel-plate (PP) geometry ( $25 \mathrm{~mm} \phi$ or 8 $\mathrm{mm} \phi$ ). The strain $\gamma$ in the parallel-plate geometry stands for the maximum strain at the edge of the plate. Except for UH850, dynamic storage and loss moduli $\left(G^{\prime}\right.$ and $G^{\prime \prime}$, respectively) as a function of the angular frequency $(\omega)$ were measured at $\gamma$ of 0.1 in the temperature $(T)$ range of $150 \sim$ $230{ }^{\circ} \mathrm{C}$, and the master curves of $\omega$-dependent $G^{\prime}$ and $G^{\prime \prime}$ were prepared. The reference temperature $\left(T_{\mathrm{r}}\right)$ for the master curves was chosen to be $190{ }^{\circ} \mathrm{C}$. The shift factor $\left(a_{T}\right)$ used to prepare the master curves was almost the same for the four samples and was well described by an single Arrhenius-type equation. For UH850 the $\omega$-dependence of $G^{\prime}$ and $G^{\prime \prime}$ was measured only at $190{ }^{\circ} \mathrm{C}$; for this polymer is categorized in so-called ultra-high molecular weight PE (UHMWPE) and therefore a very long time is required for equilibration after change of temperature. The SR measurements to obtain the relaxation modulus $G(t, \gamma)$ were made at $150{ }^{\circ} \mathrm{C}$ for T701A and SH810, and at $190{ }^{\circ} \mathrm{C}$ for PE-A, PE-B and UH850. For T701A both cone-plate and parallel-plate were used for the stress relaxation experiments and only a parallel plate geometry was employed for the stress relaxation of the other samples. The damping function $h(\gamma)$ was experimentally determined in the long time region by using Eq. (1).

\section{Results and discussion}

Fig. 1 shows the master curves of $G^{\prime}$ and $G^{\prime \prime}$ for T701A measured in the parallel-plate fixture at $190{ }^{\circ} \mathrm{C}$. This contains

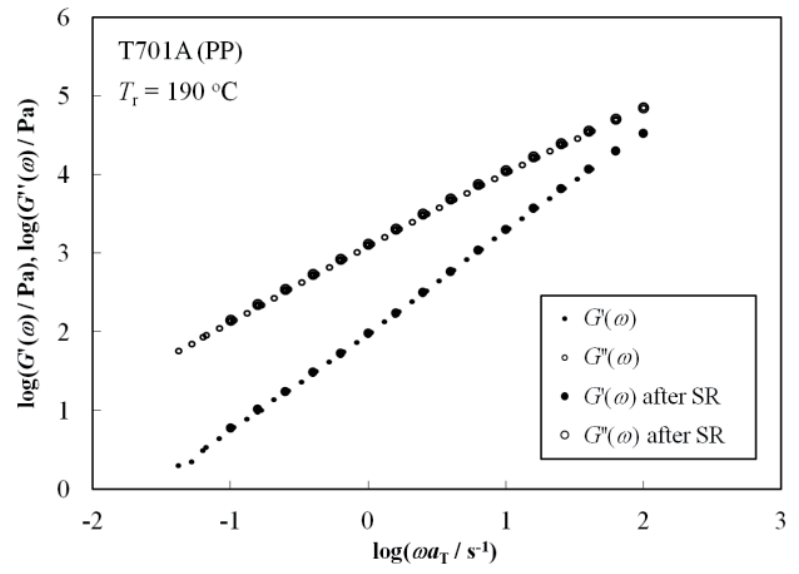

Fig. 1 Master curves of the $\omega$-dependence of dynamic storage modulus $\left(G^{\prime}\right)$ and loss modulus $\left(G^{\prime \prime}\right)$ for T701A. the data before and after SR experiments, and both sets coincide well with each other, indicating no marked change even after stress relaxation experiments requiring a long time. The figure indicates that the melt at $190{ }^{\circ} \mathrm{C}$ stays in the terminal zone. In Fig. 2 the similar plots for SH810 are shown. It is clear that the system lies in the crossover region of rubbery to terminal zone, and little effect by the SR

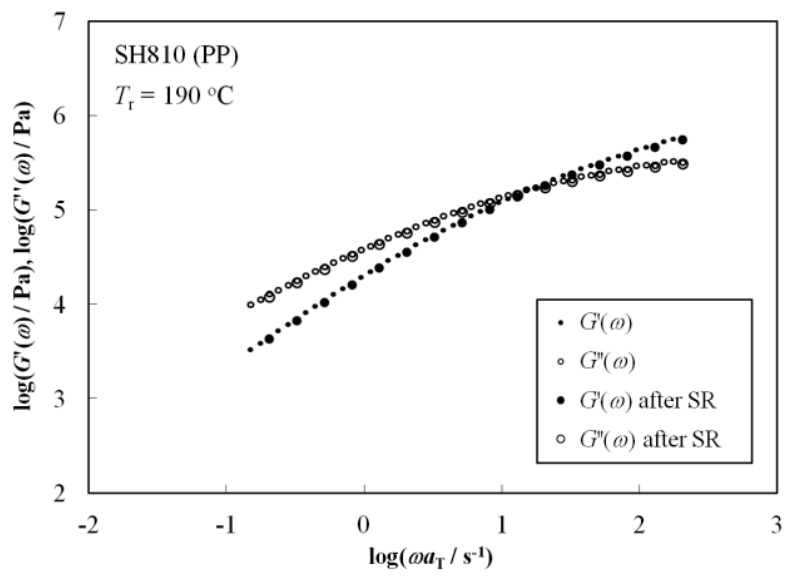

Fig. 2 Master curves of the $\omega$-dependence of dynamic storage modulus $\left(G^{\prime}\right)$ and loss modulus $\left(G^{\prime \prime}\right)$ for SH810.

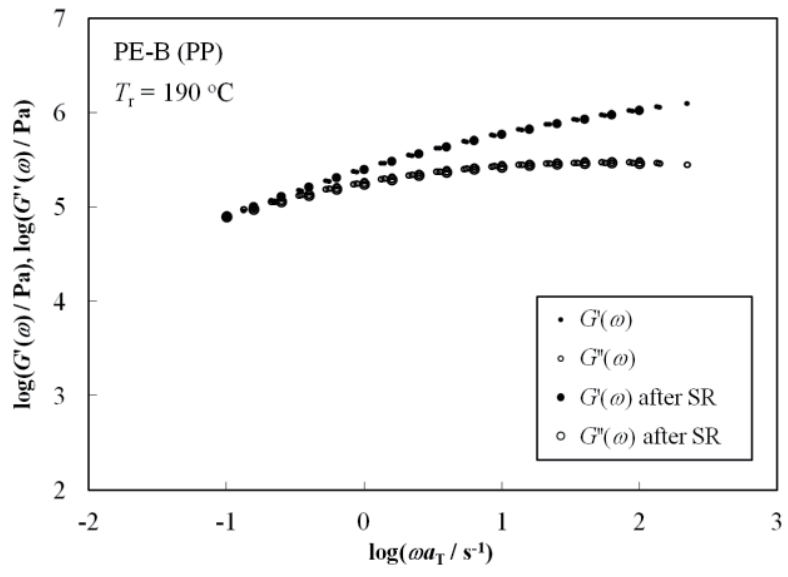

Fig. 3 Master curves of the $\omega$-dependence of dynamic storage modulus $\left(G^{\prime}\right)$ and loss modulus $\left(G^{\prime \prime}\right)$ for PE-B.

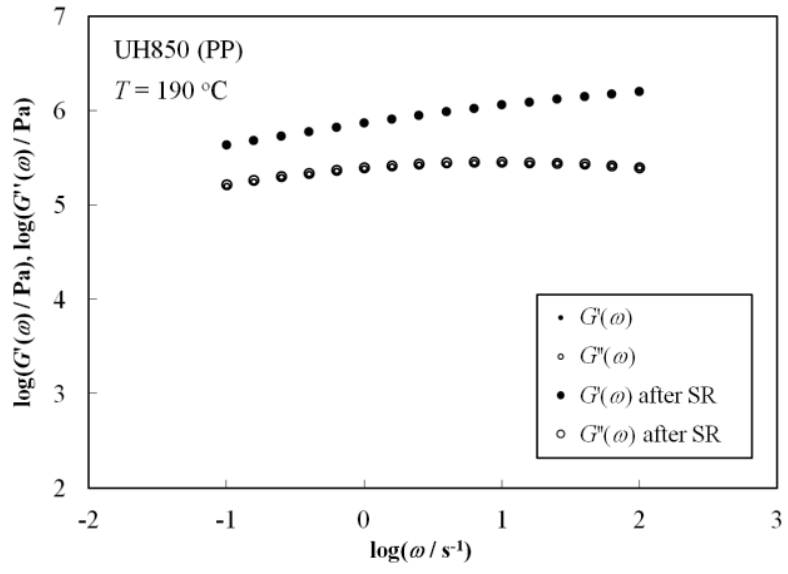

Fig. 4 Curves of the $\omega$-dependence of dynamic storage modulus $\left(G^{\prime}\right)$ and loss modulus $\left(G^{\prime \prime}\right)$ for UH850. 
experiments is observed, as is the case of T701A. Fig. 3 shows $G^{\prime}$ and $G^{\prime \prime}$ against $\omega$ plots for PE-B. The melt stays in the edge of the rubbery plateau, and there is also no marked change in the dispersion curves by the SR experiments. Similar dispersion curves of $G^{\prime}$ and $G^{\prime \prime}$ were obtained for PE-A. Fig. 4 shows the similar plots for a UHMWPE of UH850. The melt at $190^{\circ} \mathrm{C}$ lies in the rubbery plateau region and is thermally stable for a long time required for the stress relaxation measurements. Fig. 5 shows the $a_{T}$ vs. $1 / T$ plots in a semi-logarithmic scale. This contains data points of four samples (all but UH850) but they are all well approximated by a single line whose slope gives the activation enthalpy of flow $(\Delta H)$ of $30 \mathrm{~kJ} \mathrm{~mol}^{-1}$, which is identical to the value in the literature ${ }^{6}$.

Several equations have been proposed to estimate the linear relaxation modulus $G(t)$ from the dynamic viscoelastic functions. Among them we used here the following equation to estimate $G(t)$ of the PE samples ${ }^{1)}$.

$$
G(t)=\left[G^{\prime}(\omega)-0.4 G^{\prime \prime}(0.4 \omega)+0.014 G^{\prime \prime}(10 \omega)\right]_{\omega=1 / t}
$$

Fig. 6(a) shows the plots of $G(t, \gamma)$ against $t$ for T701A measured by using the parallel-pale geometry. This also contains $G(t)$ calculated by Eq. (2). The $G(t, \gamma)$ curves are almost identical in shape: they appear to be well described by a single power-law index to $t$. The $G(t, \gamma)$ curve at $\gamma=0.5$ is overlapped with the $G(t)$ curve, and as $\gamma$ increases further the $G(t, \gamma)$ curves becomes lower in position. In Fig. 6(b) the similar plots for T701A obtained in the cone-plate geometry are shown. The curves decay in a power-law manner and the power-law index is the same as that observed in Fig. 6(a), and no marked difference is seen in degree of damping between the two geometries. After determining $h(\gamma)$ how good the separability is was checked over the time region measured. Fig. 7 shows the plots of $G(t, \gamma) / h(\gamma)$ against $t$ for T701A obtained from Fig. 6(b). A single line is obtained in the whole time region, indicating that the separability holds in the entire $t$ region for $\mathrm{T} 701 \mathrm{~A}$.

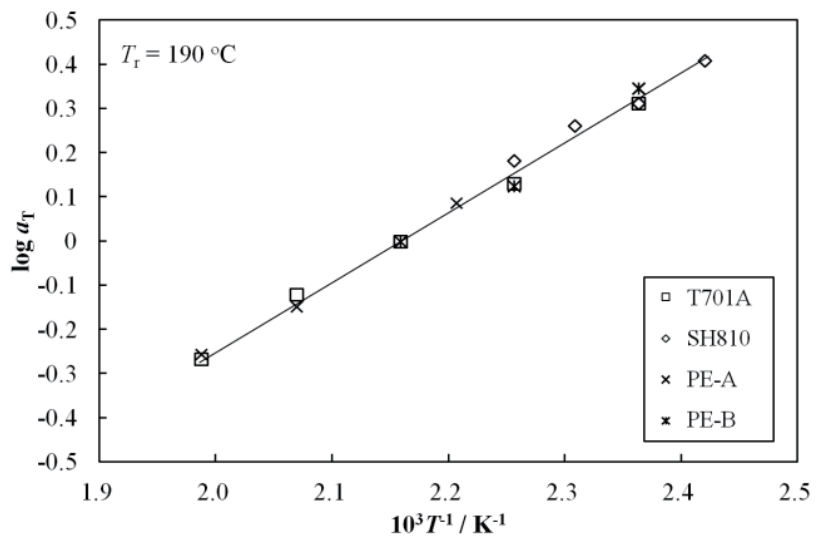

Fig. 5 Plots of the shift factor $\left(a_{T}\right)$ against $(1 / T)$ for the HDPEs.
The fixture geometry did not affect the damping behavior for T701A, but this is not always true. Isono et al. ${ }^{7)}$ showed that the damping function for the well entangled polyisobutylene obeys the DE prediction. Osaki ${ }^{4)}$ pointed out the possibility that this agreement originates from the fact that
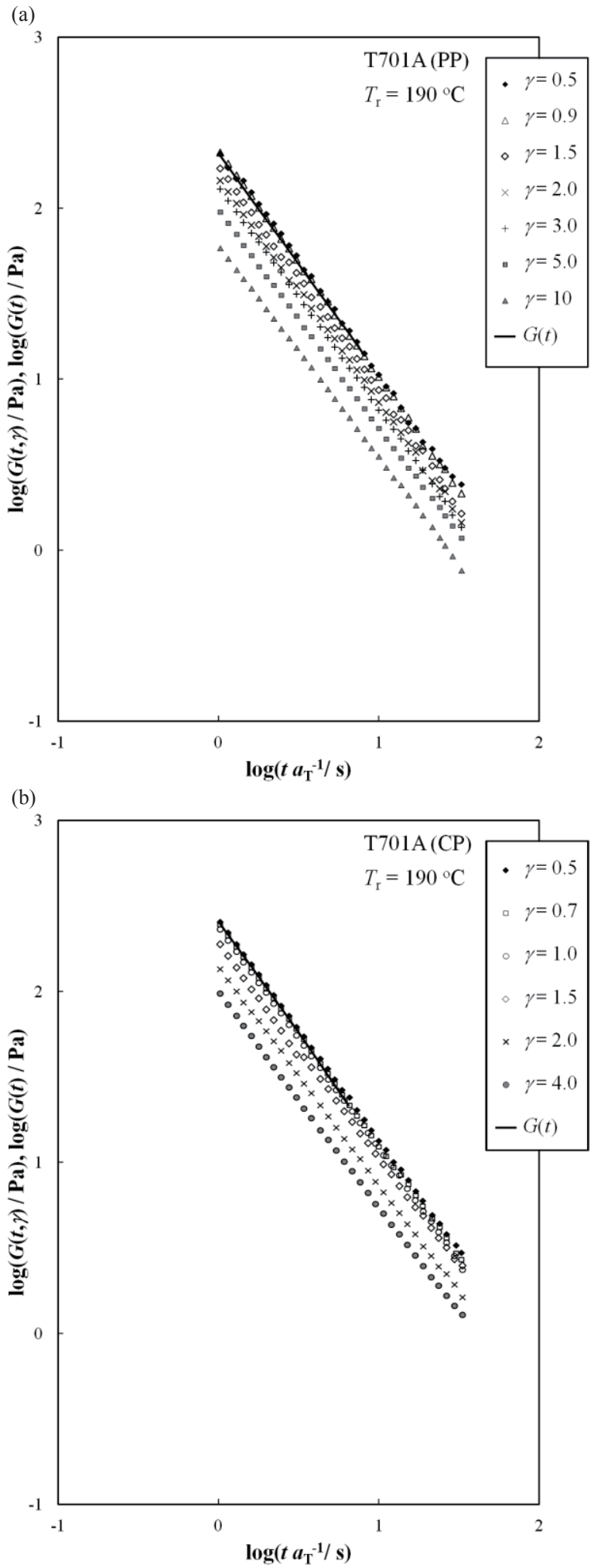

Fig. 6 The relaxation modulus $G(t, \gamma)$ plotted against $t$ for T701A. (a) for the parallel-plate geometry, (b) for the cone-plate geometry. 
they used the parallel-plate geometry. Thus, the difference in geometry appears important to examine the damping behavior Soskey and Winter ${ }^{8)}$ proposed the equation converting the damping function obtained in the parallel-plate fixture to that in the cone plate fixture. The equation can be written in the following form:

$$
h_{\mathrm{cp}}(\gamma)=h_{\mathrm{pp}}(\gamma)+\frac{\gamma}{4} \frac{d h_{\mathrm{pp}}(\gamma)}{d \gamma}
$$

where we temporary use here $h_{\mathrm{cp}}(\gamma)$ and $h_{\mathrm{pp}}(\gamma)$ for the damping functions in the cone-plate and parallel-plate geometries, respectively. This equation suggests that $h_{\mathrm{cp}}(\gamma) \leq$ $h_{\mathrm{pp}}(\gamma)$ since $d h_{\mathrm{pp}}(\gamma) / d \gamma \leq 0$. Fig. 8(a) shows $G(t, \gamma)$ plotted against $t$ for PE-B measured in the parallel-plate geometry, and in Fig. 8(b) the similar plots converted to the cone-plate geometry is shown. In both cases, the curves move downwards with increasing $\gamma$, and no marked change in slope is observed for the curves even after conversion. However, a stronger damping appears for the cone-plate geometry than for the parallel-plate geometry at large $\gamma$. This has been observed also for SH810, PE-A and UH-850. In Fig. $9 G(t, \gamma)$ / $h(\gamma)$ is plotted against $t$ by using the data in Fig. 8(b). The separability holds for the curves in the log time region, but is not good at short times for PE-B.

Fig. 10 shows the damping function $h(\gamma)$ plotted against $\gamma$ for all PE samples investigated. The converted data to the cone-plate are used for all samples except for T701A, and the

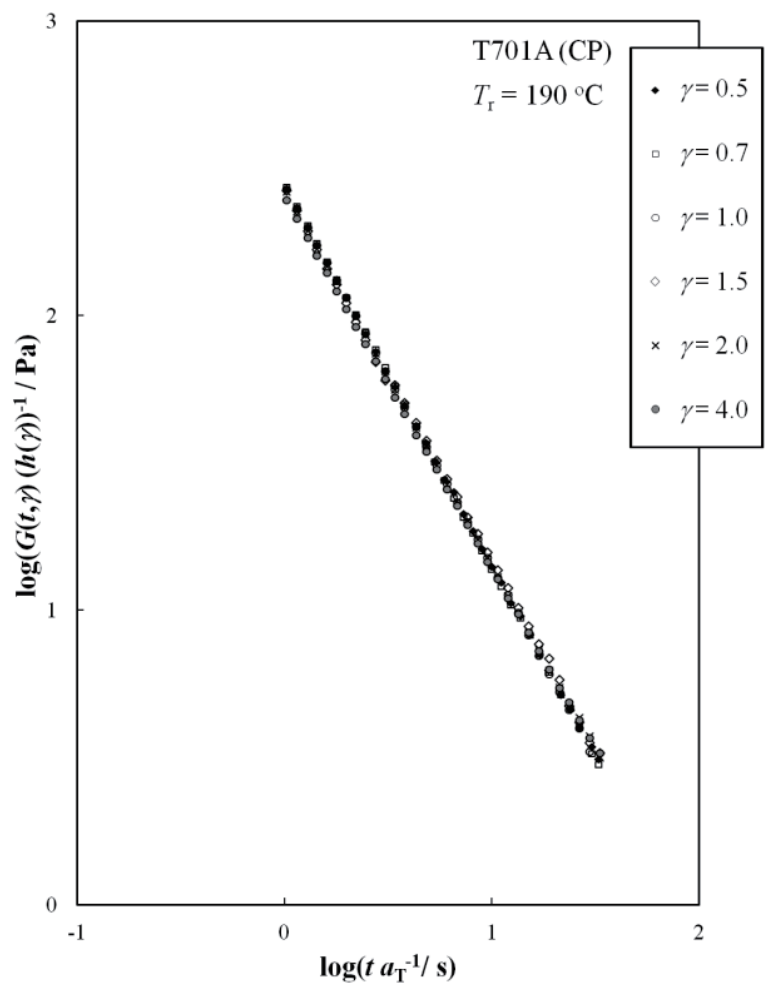

Fig. 7 The plots of $G(t, \gamma) / h(\gamma)$ vs. $t$ for T701A. data for the cone-plate geometry is adopted for T701A. The dashed curve in the figure stands for the DE prediction without use of independent alignment approximation. Only T701A shows weaker damping, i.e., weaker $\gamma$-dependence of $h(\gamma)$, than the DE prediction and the others show stronger

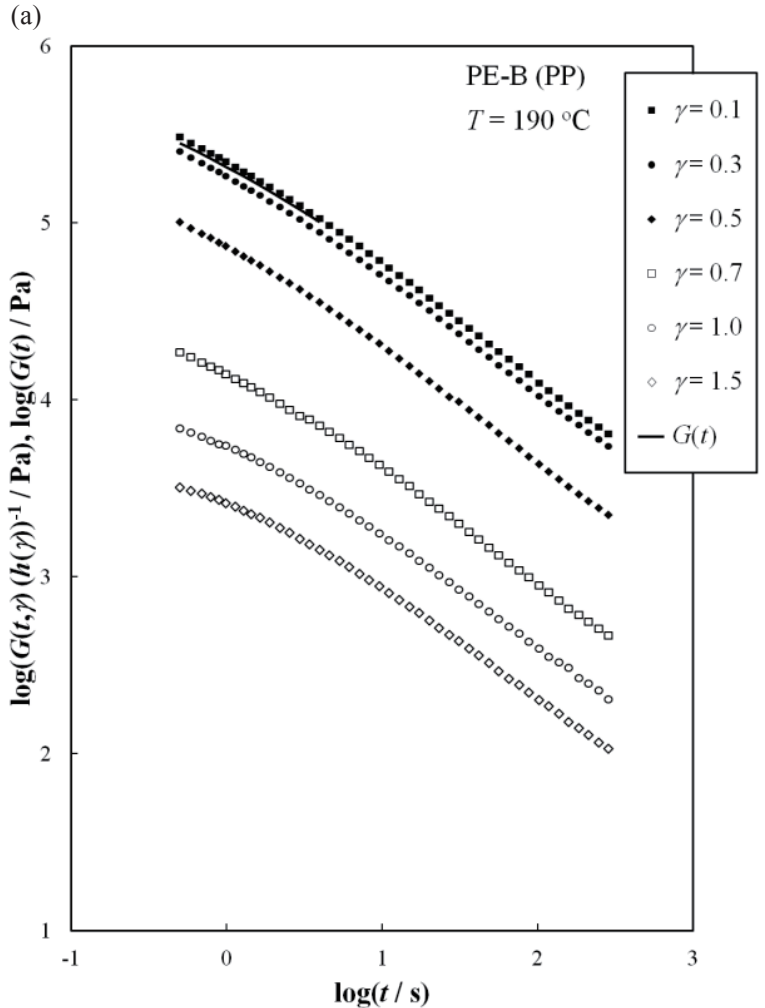

(b)

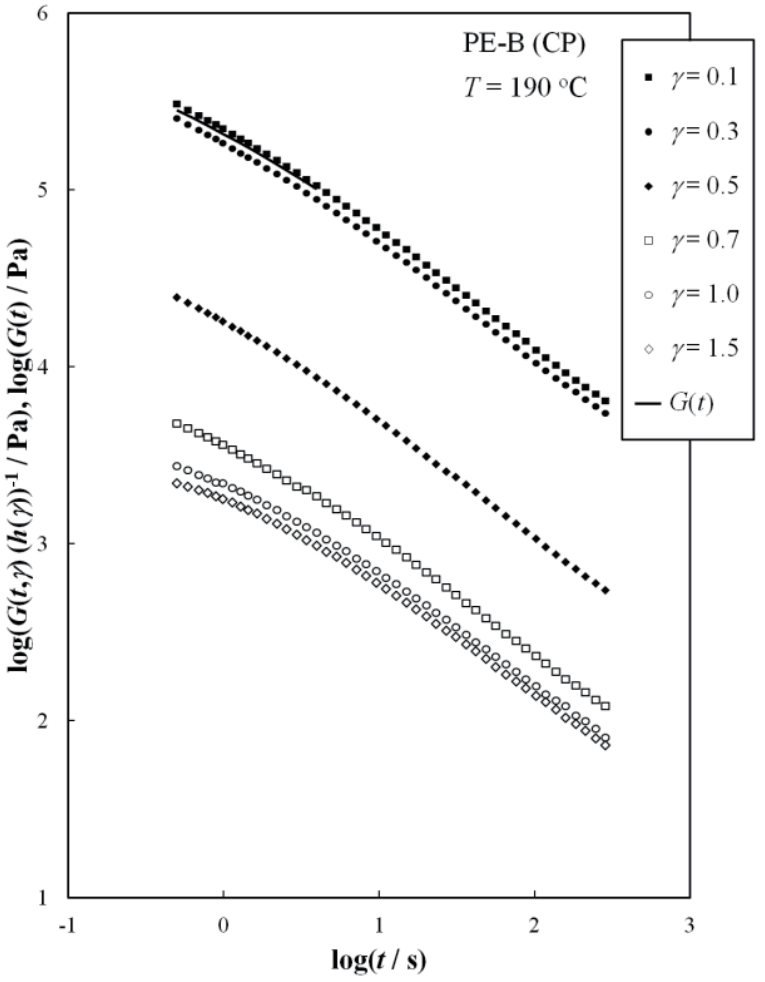

Fig. 8 The relaxation modulus $G(t, \gamma)$ plotted against $t$ for PE-B. (a) for measured in the parallel-plate geometry, (b) converted to the cone-plate geometry. 
damping. How strong $h(\gamma)$ depends on $\gamma$ is affected by several factors: the sharpness/broadness in molecular weight distribution (MWD) and the number of entanglements per chain, for example. Most of highly entangled systems with narrow MWD show the Type C behavior, where "highly" is considered to be more than 50 entanglements per chain, and the broad MWD weakens the damping ${ }^{4)}$. Seeing the molecular characteristics in Table $1, M_{\mathrm{w}} / M_{\mathrm{n}}$ lies in the range of $3.9 \sim 6.2$. This means that all samples are very broad in MWD. The value of $n_{\mathrm{e}}$ is 40 for T701A and more than 100 for the other PEs. The reason why T701A shows the weak damping is because the system is broad in MWD but does not correspond to the highly entangled system. On the other hand, the PE samples other than T701A are highly entangled

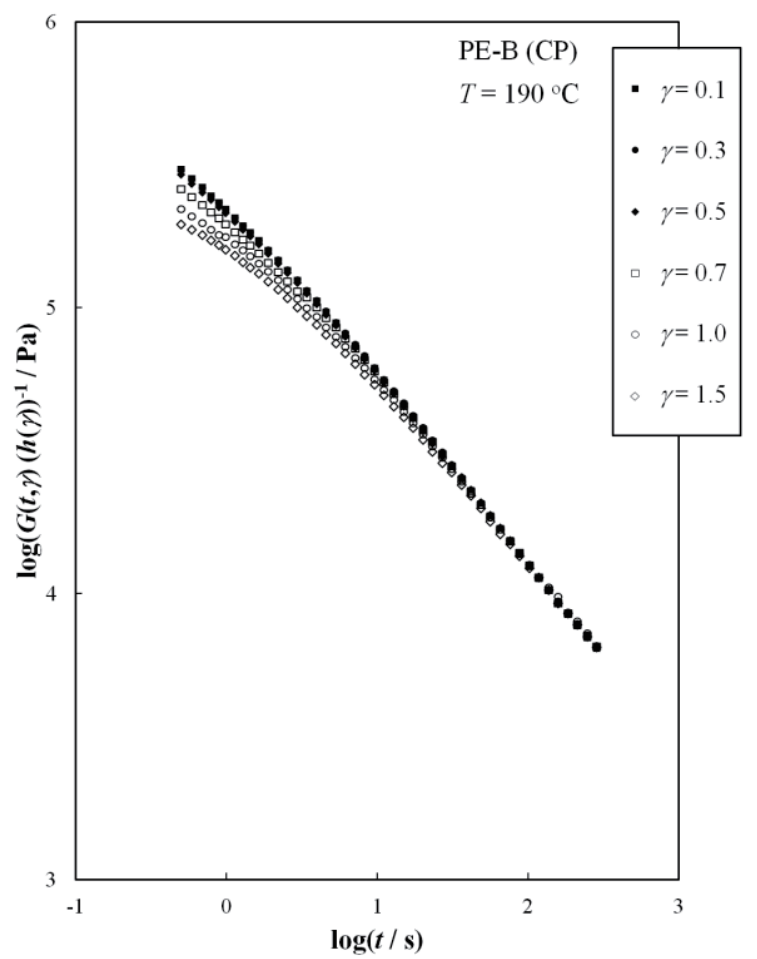

Fig. 9 The plots of $G(t, \gamma) / h(\gamma)$ vs. $t$ for PE-B (converted to the cone-plate geometry).

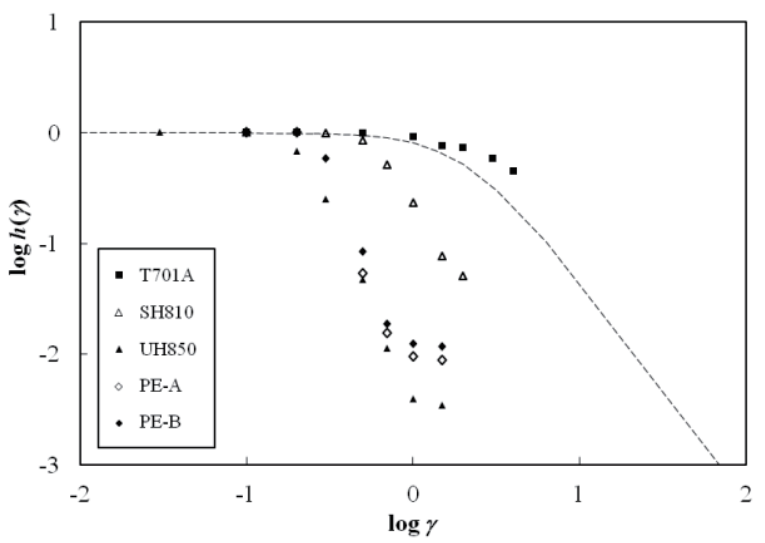

Fig. 10 The damping function $h(\gamma)$ plotted against strain $\gamma$. Dashed curve stands for the Doi-Edwards prediction. systems with broad MWD. The results in Fig. 10 indicate that the systems with broad MWD may show strong damping if the sufficient number of entanglements is formed in the systems. It is still unclear at present why the strong damping really occurs in highly entangled systems with narrow MWD. Marrucci and Grizzuti $^{5)}$ give a possible explanation for the strong damping from a theoretical viewpoint: a kind of phase separation (or mechanical instability) induced by deformation causes strong damping. When MWD is narrow such a phase separation can occur in the systems. However, when MWD is very broad the phase separation appears to be rather hard to occur. For systems with broad MWDs another cause may lie for the strong damping.

\section{Conclusion}

In this article the damping behavior was investigated for five types of HDPE samples with broader MWDs. A melt of moderately entangled HDPE shows a weaker damping than the DE prediction, maybe due to a broader MWD. The highly entangled melts show stronger damping compared with DE prediction, although the melts investigated here are very broad in MWD. The strong damping is observed for highly entangled polystyrenes with narrow MWD and is explained by a phase separation induced by deformation. For highly entangled polymers with broader MWDs another cause revealing strong damping may exist.

\section{References}

1) J. D. Ferry, "Viscoelastic properties of polymers. 3rd ed", Wiley, New York (1980).

2) H. Watanabe, "Viscoelasticity and dynamics of entangled polymers", Progress in Polymer Science, vol.24, pp.1253-1403 (1999).

3) M. Doi and S. F. Edwards, "The theory of polymer dynamics", Clarendon Press (1986) Oxford.

4) K. Osaki, "On the damping function of shear relaxation modulus for entangled polymers", Rheologica Acta, vol.32, pp.429-437 (1993).

5) G. Marrucci and N. Grizzuti, "The free energy function of the Doi-Edwards theory. Analysis of the instabilities in stress relaxation", Journal of Rheology, vol.27, pp.433-450 (1983).

6) N. Nakajima, G. A. Tirpak and M. Shida, "Branching and flow activation energy of conventional high pressure process polyethylene", Journal of Polymer Science, Polymer Letter Edition, vol.3, pp.1089-1092 (1965).

7) Y. Isono, K. Itoh, T. Komiyatani and T. Fujimoto, "Differential dynamic modulus of polyisobutylene with high molecular weight. 1 . Single-step large shearing deformations", Macromolecules, vol.24, pp.4429-4432 (1991).

8) P. R. Soskey and H. H. Winter, "Large step shear strain experiments with parallel-disk rotational rheometer", Journal of Rheology, vol.28, pp.625-645 (1984). 